\title{
Association of White matter hyperintensities and late-life depression - Insights from large scale cohort studies
}

Marvin Petersen, Benedikt M. Frey, Götz Thomalla

\section{Background and Aims}

The vascular depression hypothesis postulates that white matter hyperintensities of presumed vascular origin (WMH) may induce late-life depression via disruption of frontalsubcortical circuits involved in emotion regulation (Taylor et al., 2013; Aizenstein et al., 2016). Initially only addressed by cross-sectional studies, in the recent years more longitudinal studies started investigating in this issue. We aim to provide an overview of insights on vascular depression derived from
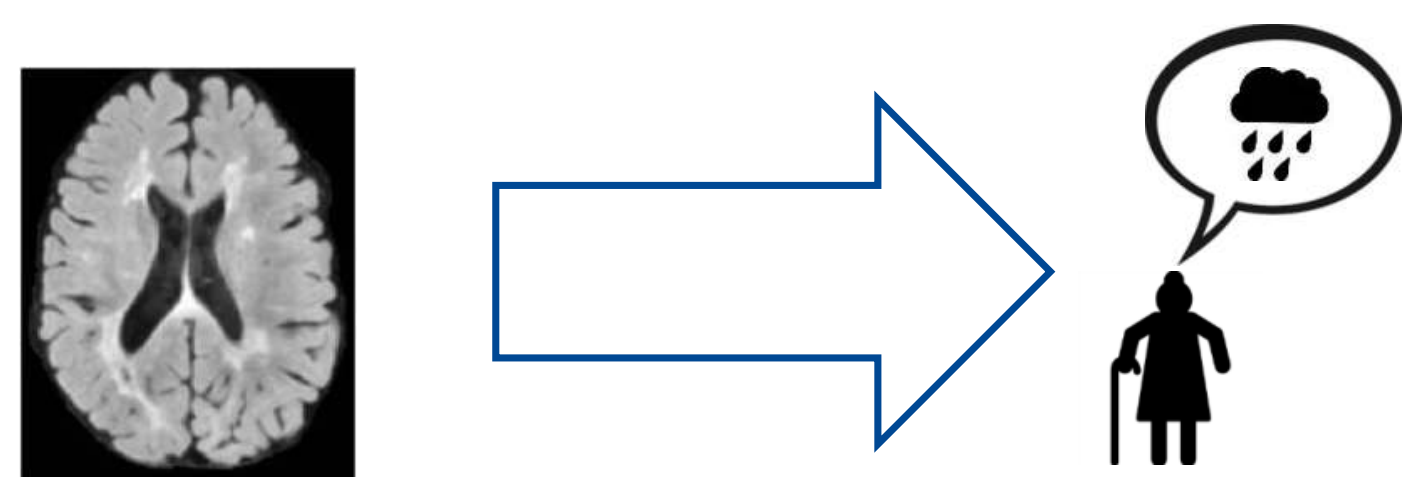

Fig 1: Vascular depression hypothesis recent prospective studies.

\section{Methods}

We performed a selective review. Articles were included if they met the following inclusion criteria: (1) investigation of the association of WMH and late-life depression, (2) data from a prospective cohort study. Data extraction was performed by full-text reading articles and standardized extraction of pre-specified information.

\section{Results}

We reviewed 15 studies from 8 different cohort studies, published from 2002 to 2015. With 5 articles the Leukoaraiosis and Disability Study (LADIS) represents the largest share. 13 studies utilized fully-quantitative WMH measurement and 5 different questionnaires (GDS, CES-D, PHQ-9, MINI, MHI-5) were applied for depression assessment. 11 studies delivered significant results for the association of WMH and late-life depression. Studies not showing significant results had lower mean sample size, a lower women proportion, their participants had lower baseline WMH volume and were younger (Tbl. 1). Studies that differentiated between WMH localizations suggest that deep WMH are more strongly associated with depressive symptom development than periventricular $\mathrm{WMH}$.

\begin{tabular}{|c|c|c|c|}
\hline & VDH + & VDH - & t-Test \\
\hline Count & 11 & 4 & \\
\hline Mean sample size & 1022,55 & 403,67 & 0,27 \\
\hline Mean age & 73,27 & 69 & 0,18 \\
\hline $\begin{array}{l}\text { Women } \\
\text { proportion }\end{array}$ & 0,52 & 0,31 & 0,020 \\
\hline $\begin{array}{c}\text { Baseline } \mathrm{WMH} \\
\text { volume }(\mathrm{ml})\end{array}$ & 9,56 & 1,50 & 0,020 \\
\hline
\end{tabular}

\section{Conclusion}

To summarize, the majority of available studies report an association of white matter hyperintensities with depression in elderly subjects and by this support the vascular depression hypothesis. Studies with contradictory findings showed methodological limitations. 